\title{
Alleviation of EGFR tyrosine kinase inhibitor-induced skin toxicity by modified Huang-Lian-Jie-Du Decoction cream in cancer patients
}

Ming-Yang Lee ${ }^{1,2 \dagger}$, Mei-Yi Lin ${ }^{3,4 \dagger}$, Yu-Ju Chang ${ }^{1}$, Yu-Ting Tseng ${ }^{3}$, I-An Huang ${ }^{3}$, Ying-Yu Siao ${ }^{5}$, Yi-Wen Liu ${ }^{5 *}$

${ }^{1}$ Departments of Hematology and Oncology, Ditmanson Medical Foundation Chiayi Christian Hospital, Chiayi 600, Taiwan

${ }^{2}$ Departments of Nursing, Min-Hwei College of Health Care Management, Tainan 736, Taiwan

${ }^{3}$ Department of Chinese Medicine, Ditmanson Medical Foundation Chiayi Christian Hospital, Chiayi 600, Taiwan

${ }^{4}$ Department of Food Nutrition and Health Biotechnology, Asian University, Taichung 41354, Taiwan

${ }^{5}$ Department of Microbiology, Immunology and Biopharmaceuticals, College of Life Sciences, National Chiayi University, Chiayi 600, Taiwan

*Corresponding author: Yi-Wen Liu, e-mail address: ywlss@ mail.ncyu.edu.tw

${ }^{\dagger}$ Ming-Yang Lee and Mei-Yi Lin contributed equally to this work 


\begin{abstract}
Background: The EGFRIs and TKIs are effective for cancer target therapy, but the acneiform rashes or called inflammatory papulopustular exanthemas are common $(50 \%$ to $100 \%$ ). The conventional therapy for EGFRIs/TKIs-induced skin toxicity is steroids and antibiotics, but their effect is still limited. In this study, a modified Chinese herbal medicine Huang Lian Jie Du decoction cream with Yin-Cold (YC) medicine characteristic was investigated for the effect on patients suffering EGFRIs/TKIsinduced skin toxicity.
\end{abstract}

Methods: The modified Huang Lian Jie Du (mHLJD) decoction cream was made from 10 herbal medicines including 4 major medicines (Huanglian, Huangqin, Huangbo, and Zhizi) in traditional HLJD decoction. Patients with EGFRIs/TKIs-induced skin toxicity were enrolled. Patients were excluded if they also used other cream for the skin toxicity. Skin conditions were follow up every 2 weeks. The patients' characteristics, the skin toxicities and treatment response were recorded and analyzed after using mHLJD decoction cream for 1 2 months.

Results: The mHLJD decoction cream and its subpackages were stored at $4{ }^{\circ} \mathrm{C}$ before use. Thirty-four patients who had grade 1-3 skin toxicities after receiving EGFRIs or TKIs were enrolled. Five patients were withdrawal or excluded, 2 patients with paronychia syndrome had no improvement and were excluded, too. Finally, data from 
27 patients were analyzed. The mean grade of rash acneiform was decreased from 2.23

(ranged 1 to 3 ) to 0.38 (ranged 0 to 1 ) after mHLJD decoction cream treatment for 1 2 months. And the mean grade of dry skin was decreased from 1.57 (ranged 1 to 2) to 0.77 (ranged 0 to 1 ). The changes of skin toxicity were significant and no obvious adverse events.

Conclusions: In summary, the mHLJD decoction cream is effective for alleviation of EGFRIs/TKIs-induced skin rash acneiform and dry skin. And mHLJD decoction cream has no effect in patients with paronychia syndrome.

Keywords: EGFRIs/TKIs, skin rash, dry skin, Chinese herbal medicine Trial registration: CYCH, CYCH-IRB No. 2019023. Registered 14 May 2019, http://www.cych.org.tw/irbweb/ 


\section{Background}

Epidermal growth factor receptor inhibitors (EGFRIs) and tyrosine kinase inhibitors (TKIs) are the popular anti-cancer drug for targeting therapy. They can be used in breast cancer, colon-rectal cancer, lung cancer, pancreatic cancer and etc. EGFRIs/TKIs inhibit the tyrosine kinase of EGFR to block EGFR-induced cancerous characteristics. Though the drugs are effective for cancer therapy, the acneiform rashes or called inflammatory papulopustular exanthemas are the most frequent (50\% to $100 \%)$ adverse event associated with the use of EGFRIs/TKIs [1]. There are two types of EGFRIs/TKIs involving monoclonal antibodies and small molecule drugs. The severe rashes occur more frequently in the former (10\%-17\%) compared with the latter (5\%9\%) [2]. Because the rash reactions affect the quality of life including infection risk increase and psychosocial activity decrease, some treatments were made to resolve the problems. Among these treatments, antibiotics and steroids are the most prescribed regimens for the treatment and prevention of EGFRIs/TKIs-induced skin reactions [3]. According to a retrospective cohort study, it indicates that an integrating dermatologic care of evidence-based prophylaxis treatment may minimize skin toxicity-associated chemotherapy interruptions [4]. Therefore, we also applied a Traditional Chinese Medicine (TCM) to evaluate relief effect on EGFRIs/TKIs-induced skin toxicity. 
The EGFRIs/TKIs-induced skin reactions including papulopustular rash, pruritus, mucositis, xerosis, fissures and etc. According to the TCM theory and the experiences of Chinese Medicine doctors, skin rashes mostly belong to the Yang-heat $(Y H)$ type of TCM syndromes. The $Y H$ type disease should be treated with Yin-Cold $(Y C)$ medicine to maintain the homeostasis of the whole body [5]. There are some TCMs could be applied as $Y C$ herbal medicine because they have been used for clearing heat, removing moisture, purge endogenous fire, toxin excretion, for example, Gan Lu Yin (GLY), Huang Lian Jie Du Tang (HLJDT), Pi Pa Qing Fei Yin (PPQFY), Tuo Li Xiao Du Yin (TLXDY), Wen Qing Yin (WQY), and Zhen Ren Huo Ming Yin (ZRHMY). They have shown some benefits for lung cancer patients with EGFR inhibitor-related dermatologic reactions [6].

The formula of HLJDT (also called HLJD decoction) was first recorded in the book "Wai-Tai-Mi-Yao" in the Chinese Tang dynasty (618-907 A.D.). It consists of four traditional herbs including Huanglian (Coptidis Rhizoma), Huangqin (Scutellariae Radix), Huangbo (Phellodendri Cortex), and Zhizi (Gardeniae Fructus) in a dry weight ratio of 3:2:2:3 (2013-150-1137_JE). HLJD decoction has been widely used in the treatment of inflammation. For example, it ameliorates type II collagen-induced rat arthritis by oral [7], reduces vascular cell adhesion molecule 1 protein expression and leukocyte-endothelial adhesion in rat lung venules after lipopolysaccharide (LPS) 
stimulation [8]. In spontaneous hypertensive rats, HLJD decoction also has antihypertensive effect and alters gene expression profile including the genes with immune function [9]. In dextran sulphate sodium-induced mice ulcerative colitis (UC), HLJD decoction alleviates the UC symptoms and decreases inflammatory cytokines in colons [10]. HLJD decoction mitigates mice atopic dermatitis induced by 2,4-dinitrochlorobenzene [11], and decreases nitric oxide and various cytokines release in LPS-stimulated RAW264.7 cells [12]. Taken together, HLJD decoction shows its effects in various inflammatory conditions.

In this study, we planned to treat EGFRIs-induced skin adverse effect by HLJD decoction but we didn't want to produce systemic action because it might interfere the anti-cancer effect of EGFRIs. Therefore, we processed the HLJD decoction as cream formulation for a tropic $Y C$ medicine. In addition to the 4 herbal medicines in original HLJD decoction, other 6 herbal medicines were also involved to make the modified HLJD (mHLJD) decoction: Sangbaipi (Mori Cortex), Longdan (Gentianae Radix et Rhizoma), Kushen (Sophorae flavescentis Radix), Tofulin (Smilacis Glabrae Rhizoma), Lianqiao (Forsythiae Fructus) and Shigao (Gypsum Fibrosum). The 6 additional herbal medicines were selected because they also have the efficacy of clearing heat ad removing moisture. 


\section{Materials and Methods}

\section{Modified HLJD (mHLJD) decoction preparation}

All the herbal medicines were purchased from Fu-Xian herbal medicine store (Chiayi, Taiwan) and were identified by a professional pharmacist I-An Hung at Ditmanson Medical Foundation Chia-Yi Christian Hospital. The dry herbal medicine included Huanglian, Huangqin, Huangbo, and Zhizi, Sangbaipi, Longdan, Kushen, Tofulin, Lianqiao and Shigao. The specimens of 10 herbal medicine were deposited at Department of Chinese Medicine of Ditmanson Medical Foundation Chia-Yi Christian Hospital.

For decoction preparation, we weighed Huanglian (168.75 g), Huangqin (112.5 g), Huangbo (112.5 g), and Zhizi (168.75 g), Sangbaipi (75 g), Longdan (37.5 g), Kushen (112.5 g), Tofulin (112.5 g), Lianqiao (75 g) and Shigao (187.5 g), mixed and put into a refined cotton bag. All the powder was socked in $4.5 \mathrm{~L}$ water for $20 \mathrm{~min}$, then decocted by boiling water for $90 \mathrm{~min}$. After taking out the cotton bag with medicines, the remaining decoction was about $3000 \mathrm{ml}$. The $\mathrm{mHLJD}$ decoction was stored at $4^{\circ} \mathrm{C}$.

\section{Process of modified HLJD (mHLJD) decoction cream for skin use}

There were 26 kinds of chemical (Table 1) in the cream preparation including the concentrated mHLJD decoction. Figure 1 shows the procedure for making mHLJD decoction cream. The mHLJD decoction cream was stored at $4{ }^{\circ} \mathrm{C}$.

\section{Patients}

Adult malignancy patients who received epidermal growth factor receptor inhibitors (EGFRIs), such as monoclonal antibodies cetuximab or panitumumab, or who received tyrosine kinase inhibitors (TKIs), such as gefitinib, erlotinib or afatinb in Ditmanson 
Medical Foundation Chia-Yi Christian Hospital were screening. The patients had 1-3 grade of skin toxicities after above medications and agreed to receive mHLJD decoction cream were enrolled after signing informed consent (IRB number of Ditmanson Medical Foundation Chia-Yi Christian Hospital: CYCH-IRB No. 2019023). The patients' age less than 20 years old, or cannot have good compliance to use topical cream to skin lesions, or has acute allergy reaction while first time use, were all excluded. When the patients received first time of mHLJD decoction cream, the topical treatment was done by physicians and acute allergy reactions was monitor for 30 minutes. After that, the patients received mHLJD decoction cream topical treatment on the skin lesions three times per day by themselves. During mHLJD treatment period, patients were excluded if they also used other cream for the skin toxicity. Skin conditions were follow up every 2 weeks. The patients' characteristics, the skin toxicities and treatment response were recorded and analyzed. The evaluation of skin toxicities was according to Common Terminology Criteria for Adverse Events (CTCAE) Version 4.0.

The managements of the side effects are according to the recommends of each drug's imitation orders. The summary of the skin toxicity management is as following table.

\begin{tabular}{|l|l|l|}
\hline Skin adverse effects grading & EGFRI / TKI drugs & mHLJD decoction cream \\
\hline Grade 1 & Original dose & Keep treatment \\
\hline Grade 2 & Original dose & Keep treatment \\
\hline Grade 3, tolerable side effect & Original dose & Keep treatment, stop if no \\
\hline
\end{tabular}




\begin{tabular}{|l|l|l|}
\hline & & improvement within 7 days \\
\hline Grade 3, intolerable side effect & Stop for one week & Keep treatment, stop if no \\
& or & improvement within 7 days \\
\hline Grade 4 & Reduced dose & Keep treatment, stop if no \\
& Stop treatment & improvement within 3 days \\
\hline
\end{tabular}

\section{Statistical Analysis}

The Statistical Package of Social Sciences software (Version 16.01; SPSS, Inc., Chicago, IL) was used for statistical analysis. The Chi-square or Fisher exact was used for testing dichotomous variables. The level of statistical significance was set at $<0.05$. 


\section{Results}

From June 2019 to April 2020, 34 adult patients who had skin toxicities after receiving EGFRIs or TKIs were enrolled. The initial characterizes of skin toxicities were included as following: rash acneiform, dry skin, scalp pain, pruritus, pain of skin, palmar-plantar erythrodysesthesia, skin ulcer, and paronychia.

Among the 34 patients, one case withdrew the study 1 week later due to uncomfortable sticky sensation after smearing mHLJD decoction cream. Another one case withdrew the study due to refuse regularly follow up in our out-patient clinic. In addition, there are 3 cases were also excluded in final analysis because two of them used other topical cream by themselves and another one case stopped TKI treatment due to cancer disease progression after enroll the study. Furthermore, two cases had paronychia as initial skin side-effect and did not have any improvement after using mHLJD decoction cream treatment for 1 week. These two cases changed to use other topical medications and withdrew this study. The procedure was summarized in Figure 2. For the fine tuning, we then added paronychia as one of exclusion criteria after group discussion. Therefore, total 27 cases successfully received more than 1 month mHLJD decoction cream treatment can be analyzed about treatment response. During the mHLJD decoction cream treatment, the EGFRIs/TKIs treatment were kept the same dosage except 2 sever cases with intolerance grade 3 skin toxicity. In these 2 cases, the TKI (erlotinib) was paused for one week, but mHLJD decoction cream treatment continued. The basic information and EGFRIs or TKIs receiving of these 27 patients was in Table 2. The incidence percentage and the most severe grade of the skin toxicities about these 27 cases were summarized in Table 3.

After mHLJD decoction cream treatment, the skin toxicities had improvement generally. The most common skin toxicities in our study is rash acneiform $(n=26$, 
96.30\%). Calculated the most severe grade of every patient, the mean grade of rash acneiform is 2.23 (ranged 1 to 3 ). After treatment for a period (1 2 months, depends on cases), the mean grade of rash acneiform is 0.38 (ranged 0 to 1 ). The improvement is significant $(\mathrm{p}<0.001)$. The grading change of rash acneiform after mHLJD decoction cream treatment is presented as figure 3.

The second common skin toxicities in our study is dry skin $(n=7,25.93 \%)$. Record the most severe grade of every patient and calculate the mean grade of dry skin is 1.57 (ranged 1 to 2 ). After treatment for a period (1 2 months), the mean grade of dry skin is 0.77 (ranged 0 to 1 ). The improvement is also significant $(\mathrm{p}=0.002)$. The grading change of dry skin after mHLJD decoction cream treatment in each patient is presented as figure 4 .

Figure 5 shows the photos for a patient with rash acneiform on his back. He was enrolled after $400 \mathrm{mg}$ cetuximab treatment for about one month. The rash acneiform was grade 3 before mHLJD decoction cream treatment (figure 5A). After smearing mHLJD decoction cream every day, the grade decreased to 0 with only scars on his back (figure 5B). The photos in figure 6 are from a patient with $40 \mathrm{mg}$ afatinib treatment She was enrolled after afatinib treatment for 2 weeks. The rash acneiform on his face was grade 3 (figure 6A). After mHLJD decoction cream treatment for about 1 month, the grade decreased to 0 (figure 6B), and this skin condition became better after four months later (figure 6C) and seven months later (figure 6D) without stop or decrease afatinib dosage. Another special case is in figure 7 with rash acneiform on her legs. She was enrolled after $150 \mathrm{mg}$ erlotinib treatment for about 3 months. After mHLJD decoction cream treatment for about 1 month, the rash acneiform was decreased from grade 2 (figure 7A) to grade 1 (figure 7B). Actually, the skin condition was getting 
better after two more months (figure 7C) and five months later (figure 7D) without anticancer regimen change. 


\section{Discussion}

In Taiwan, Chinese Medicine clinic is very popular in each city and is essential in a larger hospital. In Ditmanson Medical Foundation Chia-Yi Christian Hospital, Chinese Medicine clinics have their own patients, they also serve consultations for other departments, for example, cancer treatment in hematology oncology. Recently, the cancer patients accepting molecular targeting drug therapy are gradually increase, especially EGFRIs and TKIs. As the skin toxicity induced by EGFRIs and TKIs is getting more serious and limited improvement by steroids and antibiotic treatment, the alternative therapy by Chinese Medicine is introduced and get a significant improvement in this study.

According to the Chinese therapy theory, HLJD decoction might have the ability to relieve the EGFRIs/TKIs-induced skin toxicity. In this study, mHLJD decoction cream really shows a significant effect on relieving EGFRIs/TKIs-induced rash acneiform (Figure 3) and dry skin (Figure 4). In addition to the Chinese theory, some chemicals in the herbal medicine have been reported to have their biological function related to anti-skin inflammation. The major chemical in Huanglian and Huangbo is berberine. Berberine has been reported to produce anti-inflammatory effect in human keratinocytes, for example, it inhibits 12-O-tetradecanoylphorbol-13-acetate (TPA)induced matrix metalloproteinase- 9 activity and interleukin-6 expression which are indicators of aging and inflammation [13]. It also inhibits heatkilled Propionibacterium acnes-induced nitric oxide and proinflammatory cytokine production in $\mathrm{HaCaT}$ keratinocytic cells via inhibiting mitogen-activated protein kinase and NF- $\kappa \mathrm{B}$ signaling pathways [14].

The major chemicals in Huangqin are baicalin, baicalein and wogonin. In a mice study, it suggests that baicalin can be metabolized to baicalein and oroxylin A by 
intestinal microflora. All these compounds inhibit lipopolysaccharide (LPS)-stimulated peritoneal cytokine production and NF-kB activation in mice [15]. Baicalein also inhibits TPA-induced skin infiltration of polymorphonuclear leukocytes in mice dermis [16]. Wogonin, a derivative of baicalein, also inhibits IL-1beta or TNF-induced cyclooxygenase-2 expression in skin fibroblast NIH/3T3 cells [17].

The major compound in Zhizi is geniposide. It has been reported that geniposide has anti-inflammation effect in LPS-activated rat fibroblast-like synoviocytes [18]. It also has anti-inflammation effect in LPS-induced mice mastitis [19]. Furthermore, geniposide can be absorbed by skin and accumulated in subcutaneous tissue within $1 \mathrm{~h}$ in a mice study [20]. In addition to anti-inflammation effect of berberine, baicalin and geniposide, berberine also has anti-bacterial activity [21, 22]. Therefore, the potential anti-inflammation and anti-bacterial effects of these compounds in mHLJD decoction cream might constitute of the therapeutic effect in this trial.

In previous studies, the pathophysiology of EGFRIs/TKIs-induced paronychia includes disruption of the protective barrier between the nail and the nail fold, and secondary bacterial and fungal infections $[23,24]$. The majority compounds of mHLJD decoction cream, such like berberine, have the anti-bacterial effect but focus on Staphylococcus [25]. However, the bacterial specimens of paronychia can be both aerobic bacteria (for example Staphylococcus aureus) and anaerobic bacteria [26, 27], 
thus in our study, it may be the reason that mHLJD decoction cream couldn't have good therapeutic effect for paronychia lesions.

\section{Conclusions}

In this study, we conclude that the mHLJD decoction cream is effective for EGFRIs/TKIs-induced skin rash acneiform and dry skin. We hope a better adverse effect control will keep the anti-cancer drug dosage and have a good anti-cancer therapy effect. 


\begin{abstract}
Abbreviations
EGFRIs: epidermal growth factor inhibitors; TKIs: tyrosine kinase inhibitors; mHLJD: modified Huang Lian Jie Du; TCM: Traditional Chinese Medicine; YH: Yang-heat; YC: Yin-Cold; GLY: Gan Lu Yin; HLJDT: Huang Lian Jie Du Tang; PPQFY: Pi Pa Qing Fei Yin; TLXDY: Tuo Li Xiao Du Yin; WQY: Wen Qing Yin; ZRHMY: Zhen Ren Huo Ming Yin; LPS: lipopolysaccharide; IRB: Institutional Review Board; TPA: 12$O$-tetradecanoylphorbol-13-acetate.
\end{abstract}

\title{
Declarations
}

\section{Ethics approval and consent to participate}

The mHLJD decoction cream clinical trial was approved by the Institutional Review Board of the Ditmanson Medical Foundation Chia-Yi Christian Hospital (CYCH-IRB No. 2019023).

\section{Consent for publication}

Not applicable.

\section{Availability of data and materials}

The datasets used in the current study are available from the corresponding author on reasonable request. 


\section{Competing interests}

The authors declare that they have no competing interests.

\section{Funding}

This study was supported by Ditmanson Medical Foundation Chia-Yi Christian Hospital Research Program R108-014 and the Ministry of Science and Technology, Taiwan, R.O.C. (MOST108-2320-B-415-006-MY3).

\section{Authors' contributions}

MY Lee, MY Lin and YW Liu conceived and designed the study. MY Lee, YJ Chang and $\mathrm{Yu}$ Chang cared and recorded the patients' condition. IA Huang and YY Siao prepared the mHLJD decoction. MY Lin and YW Liu arranged the cream preparation. MY Lee, MY Lin and YW Liu analyzed the data and wrote the paper. All authors read and approved the final manuscript.

\section{Acknowledgements}

Not applicable. 


\section{References}

1. Fabbrocini G, Panariello L, Caro G, Cacciapuoti S: Acneiform Rash Induced by EGFR Inhibitors: Review of the Literature and New Insights. Skin Appendage Disord 2015;(1):31-7.

2. Lacouture ME, Anadkat MJ, Bensadoun RJ, Bryce J, Chan A, Epstein JB, EabySandy B, Murphy BA, Group MSTS: Clinical practice guidelines for the prevention and treatment of EGFR inhibitor-associated dermatologic toxicities. Support Care Cancer 2011;19(8):1079-95.

3. Kozuki T: Skin problems and EGFR-tyrosine kinase inhibitor. Jpn J Clin Oncol 2016;46(4):291-8.

4. Yu Z, Dee EC, Bach DQ, Mostaghimi A, LeBoeuf NR: Evaluation of a Comprehensive Skin Toxicity Program for Patients Treated With Epidermal Growth Factor Receptor Inhibitors at a Cancer Treatment Center. JAMA Dermatol 2020. (Online ahead of print)

5. Hung YC, Chin CY, Lee YC, Chen YH, Tsai MY: Clinical experience of chinese herbal medicine ameliorates dermatologic events from epidermal growth factor receptor inhibitors for lung cancer: A case series. Explore (NY) 2019;15(5):36370.

6. Zhu YJ, Zhang HB, Liu LR, Liu YH, Zhang FL, Bai JP, Li Y, Qu YC, Qu X, Chen $\mathrm{X}$ et al: Yin-Cold or Yang-Heat Syndrome Type of Traditional Chinese Medicine Was Associated with the Epidermal Growth Factor Receptor Gene Status in Non-Small Cell Lung Cancer Patients: Confirmation of a TCM Concept. Evidence-Based Complementary and Alternative Medicine 2017;2017:Article ID 7063859.

7. $\mathrm{Hu} \mathrm{Y,} \mathrm{Hu} \mathrm{Z,} \mathrm{Wang} \mathrm{S,} \mathrm{Dong} \mathrm{X,} \mathrm{Xiao} \mathrm{C,} \mathrm{Jiang} \mathrm{M,} \mathrm{Lv} \mathrm{A,} \mathrm{Zhang} \mathrm{W,} \mathrm{Liu} \mathrm{R:}$ Protective effects of Huang-Lian-Jie-Du-Tang and its component group on collagen-induced arthritis in rats. J Ethnopharmacol 2013;150(3):1137-44.

8. Wu YH, Chuang SY, Hong WC, Lai YJ, Chang YL, Pang JH: In vivo and in vitro inhibitory effects of a traditional Chinese formulation on LPS-stimulated leukocyte-endothelial cell adhesion and VCAM-1 gene expression. J Ethnopharmacol 2012;140(1):55-63.

9. Yue GH, Zhuo SY, Xia M, Zhang Z, Gao YW, Luo Y: Effect of huanglian jiedu decoction on thoracic aorta gene expression in spontaneous hypertensive rats. Evid Based Complement Alternat Med 2014;2014:565784.

10. Lu Z, Xiong W, Xiao S, Lin Y, Yu K, Yue G, Liu Q, Li F, Liang J: Huanglian Jiedu Decoction ameliorates DSS-induced colitis in mice via the JAK2/STAT3 signalling pathway. Chin Med 2020;15:45.

11. Fan HJ, Zhao XS, Tan ZB, Liu B, Xu HL, Wu YT, Xie LP, Bi YM, Lai YG, 
Liang HF et al: Effects and mechanism of action of Huang-Lian-Jie-Du-Tang in atopic dermatitis-like skin dysfunction in vivo and in vitro. J Ethnopharmacol 2019;240:111937.

12. Chen Y, Xian Y, Lai Z, Loo S, Chan WY, Lin ZX: Anti-inflammatory and antiallergic effects and underlying mechanisms of Huang-Lian-Jie-Du extract: Implication for atopic dermatitis treatment. J Ethnopharmacol 2016;185:41-52.

13. Kim S, Kim Y, Kim JE, Cho KH, Chung JH: Berberine inhibits TPA-induced MMP-9 and IL-6 expression in normal human keratinocytes. Phytomedicine 2008;15(5):340-7.

14. Lee JW, Kang YJ, Choi HK, Yoon YG: Fractionated Coptis chinensis Extract and Its Bioactive Component Suppress Propionibacterium acnes-Stimulated Inflammation in Human Keratinocytes. J Microbiol Biotechnol 2018;28(6):839-48.

15. Jung MA, Jang SE, Hong SW, Hana MJ, Kim DH: The role of intestinal microflora in anti-inflammatory effect of baicalin in mice. Biomol Ther (Seoul) 2012;20(1):36-42.

16. Ma GZ, Liu CH, Wei B, Qiao J, Lu T, Wei HC, Chen HD, He CD: Baicalein inhibits DMBA/TPA-induced skin tumorigenesis in mice by modulating proliferation, apoptosis, and inflammation. Inflammation 2013;36(2):457-67.

17. Chi YS, Kim HP: Suppression of cyclooxygenase-2 expression of skin fibroblasts by wogonin, a plant flavone from Scutellaria radix. Prostaglandins Leukot Essent Fatty Acids 2005;72(1):59-66.

18. Deng R, Li F, Wu H, Wang WY, Dai L, Zhang ZR, Fu J: Anti-inflammatory Mechanism of Geniposide: Inhibiting the Hyperpermeability of Fibroblast-Like Synoviocytes via the RhoA/p38MAPK/NF-kappaB/F-Actin Signal Pathway. Front Pharmacol 2018;9:105.

19. Song X, Zhang W, Wang T, Jiang H, Zhang Z, Fu Y, Yang Z, Cao Y, Zhang N: Geniposide plays an anti-inflammatory role via regulating TLR4 and downstream signaling pathways in lipopolysaccharide-induced mastitis in mice. Inflammation 2014;37(5):1588-98.

20. Wang Y, Li L, Li H, Zhu Z, Hua L, Lei F, Kheir MM, Du L: Transdermal permeation of geniposide in the herbal complex liniment in vivo and in vitro. Int J Pharm 2010;392(1-2):72-7.

21. Chu M, Zhang MB, Liu YC, Kang JR, Chu ZY, Yin KL, Ding LY, Ding R, Xiao RX, Yin YN et al: Role of Berberine in the Treatment of Methicillin-Resistant Staphylococcus aureus Infections. Sci Rep 2016;6:24748.

22. More NV, Kharat KR, Kharat AS: Berberine from Argemone mexicana L exhibits a broadspectrum antibacterial activity. Acta Biochim Pol 
2017;64(4):653-60.

23. Holcmann M, Sibilia M: Mechanisms underlying skin disorders induced by EGFR inhibitors. Mol Cell Oncol 2015;2(4):e1004969.

24. Shafritz AB, Coppage JM: Acute and chronic paronychia of the hand. J Am Acad Orthop Surg 2014;22(3):165-74.

25. Tan J, Wang J, Yang C, Zhu C, Guo G, Tang J, Shen H: Antimicrobial characteristics of Berberine against prosthetic joint infection-related Staphylococcus aureus of different multi-locus sequence types. BMC Complement Altern Med 2019;19(1):218.

26. Whitehead SM, Eykyn SJ, Phillips I: Anaerobic paronychia. Br J Surg 1981;68(6):420-2.

27. Rockwell PG: Acute and chronic paronychia. Am Fam Physician 2001;63(6):1113-6. 


\section{Figure legends}

Figure 1. The procedure of mHLJD decoction cream preparation.

Figure 2. The procedure of 34 enrolled patients to 27 analyzed cases.

Figure 3. The grade change of rash acneiform after mHLJD decoction cream treatment in the 26 patients. The individual grade difference before and after MHLJD decoction cream treatment.

Figure 4 . The grade change of dry skin after mHLJD decoction cream treatment in the 7 patients. The individual grade difference before and after mHLJD decoction cream treatment.

Figure 5. One case of cetuximab-induced rash acneiform. (A) The rash acneiform before mHLJD decoction cream treatment. (B) After mHLJD decoction cream treatment for 1 month.

Figure 6. One case of afatinib-induced rash acneiform. (A) The rash acneiform before mHLJD decoction cream treatment. (B) After mHLJD decoction cream treatment for 1 
month. (C) After mHLJD decoction cream treatment for about 5 months. (D) After mHLJD decoction cream treatment for about 8 months.

Figure 7. One case of erlotinib-induced rash acneiform. (A) The rash acneiform before mHLJD decoction cream treatment. (B) After mHLJD decoction cream treatment for about 1 month. (C) After mHLJD decoction cream treatment for about 3 months. (D) After mHLJD decoction cream treatment for about 6 months. 